\title{
Pressure distribution in tilting and reclining wheelchairs with an air cushion: A pilot study
}

\author{
Hyunwoo Choi, Heymin Lee, Jeongwon Choi, Jisu Moon, Juhyang Jeong, Dohee Joo, Ingyu Yoo
}

Department of Occupational Therapy, College of Medical Science, Jeonju University, Republic of Korea

\begin{abstract}
Study aim: The aim of this study was to determine the optimal angle for maximizing pressure distribution in two types of wheelchairs (tilting and reclining) while using a ROHO cushion, which offers relatively effective pressure distribution.

Material and methods: This study enrolled 28 male and female college students who understood the purpose of the study and agreed to participate. This study aimed to determine the optimal angle for tilting and reclining wheelchairs when using a ROHO cushion at angles of $10^{\circ}, 20^{\circ}$, and $30^{\circ}$ with tilted wheelchairs and $90^{\circ}, 110^{\circ}$, and $130^{\circ}$ with reclining wheelchairs.

Results: The analysis showed that an improved pressure distribution when a tilting wheelchair was used versus a reclining one. A reclining position of $\geq 110^{\circ}$ and a tilt angle of $\geq 20^{\circ}$ led to significant pressure-reducing effects.

Conclusion: The results of this study will help wheelchair users or their guardians to select the optimal wheelchair angle when changing their posture to prevent bedsores. Although no slippage was observed in our study, it is important to remember the proper back position to prevent unnecessary slippage.
\end{abstract}

\section{Keywords: Cushion - Pressure sore - Wheelchair}

\section{Introduction}

Wheelchairs facilitate access by users in the community. However, according to the 2011 Wheelchair Safety Accident Survey Report, $65.2 \%$ of users reported that they experienced pressure sores due to sitting for a long time [6].

Pressure sores are a condition in which constant pressure on certain areas of the body disrupts the circulation of oxygen and nutrients to these areas. If severe, this can cause necrosis. The risk of pressure sores is increased if proper pressure distribution over the buttocks is not achieved when the individual is seated in the wheelchair $[15,20]$. The main cause of pressure sores is increased pressure through prolonged sitting in the wheelchair. In particular, when nerve tissues or sensory functions are impaired, buttock bedsores due to long-term use of the wheelchair may not be recognized in time, leading to necrosis. The development of pressure ulcers has negative impacts on the patient's prognosis [9].

The basic principle for preventing pressure sores is to reduce the constant pressure while seated in the wheelchair [10]. The most common mode of adjustment based on this principle is, first, to reduce the area exposed to high pressure by distributing the pressure over the contact surface.
This is especially true for patients with major limitations who have difficulty changing their posture independently [1]. Pressure on the sores is reduced by dispersing the overall pressure over the contact surface. Cushions designed to prevent pressure sores have been developed in various forms and involve the use of air, marbles, T-forms, blends, and gels, among others. Among them, the type with the highest level of user satisfaction is the air cushion [9]. This was supported by a study on the guidelines for the prevention of bed sores [21] and a comparative study on sitting pressure distribution according to the type of cushion for preventing bedsores [8]. Other studies, such as an analysis of body pressure distribution according to the design elements of the cushioning air cell, have shown that the pneumatic cushion is more effective compared to the other types $[2,5,14,21]$.

Second, wheelchair tilt can be adjusted to reduce the pressure. There are two types of wheelchairs: the tilting type, in which the seat and back are rotated backward together, and the reclining type, in which only the back support can be laid back. Past studies have shown that both tilting and reclining adjustments can reduce excessive pressure on the buttocks during wheelchair use. This has been suggested as a particularly useful intervention for patients with quadriplegia [13]. 
However, despite various studies on wheelchairs and the development of bedsore-prevention cushions, many users with spinal cord and other injuries still suffer from bedsores [11]. A good posture is one from which the individual can function optimally, which means a posture from which one can respond appropriately to various situations with minimal energy consumption [3]. Appropriate seating for wheelchair users facilitates user stability and normal sensory input to enable efficient interaction with the surrounding environment, suppresses discomfort caused by abnormal sensations, and reduces the risk of bedsores due to pressure overload [14].

In this regard, two important studies were identified. First, regarding the adjustment of the wheelchair backrest angle, previous studies have suggested a tilt angle of $20^{\circ}$ or more for the relief of hip joint pressure [25] and a reclining angle of $120^{\circ}$ or more. Another study compared the average pressure index according to the type of cushion and the angle of the wheelchair and showed effective reduction in sitting pressure [13]. The results of each study were specific to the individuals studied, and each reported limited success in reaching an integrated conclusion $[11,25]$.

Using a cushion is the easiest way to prevent bedsores. The cushion is employed to prevent bedsores from occurring before they develop and serves here as a representative intervention method to reduce sitting contact pressure [1,2]. This study was based on the hypothesis that pressure distribution would be more effective if, in addition to using a cushion, the angle of the wheelchair were properly adjusted.

This study was carried out to determine the optimal angle for maximizing the pressure distribution with two types of wheelchairs, tilting and reclining, while using a $\mathrm{ROHO}$ cushion, which offers relatively effective pressure distribution.

\section{Material and methods}

\section{Participants}

This study was conducted with 28 college students (14 males and 14 females; mean age 23.7 years, mean height $167.57 \mathrm{~cm}$, mean weight $61.12 \mathrm{~kg}$, hip width 32.0 $\mathrm{cm}$, mean femoral length $45.5 \mathrm{~cm}$ ) who understood the purpose of the study and agreed to participate. All subjects were provided with enough information to understand the experiment, after which they agreed to participate in the study. The inclusion criteria were as follows.

First, we recruited healthy men and women in their $20 \mathrm{~s}$ who met the age-appropriate criteria for healthy 20 24-year-olds in the 2015 Korea Body Size Survey Index (height: male $174.2 \mathrm{~cm}$, female $160.9 \mathrm{~cm}$; weight: male $71.5 \mathrm{~kg}$, female $55.1 \mathrm{~kg}$; hip width: male $32.8 \mathrm{~cm}$, female $32.5 \mathrm{~cm}$; thigh length: male $30.3 \mathrm{~cm}$, female $28.6 \mathrm{~cm}$ ) [23]. Second, they had no experience with pressure measurement. Third, they could maintain their posture for repeated measurements. Fourth, they had no musculoskeletal disorders (upper or lower limbs). The experiment was approved by the Institutional Review Board of Jeonju University.

\section{Measurements}

\section{Air cushion}

We used a ROHO air cushion (MK-ASC-5B, Mickey Korea), which is mainly used to prevent bedsores. The cushion is designed in the form of a closed air grid, with airflow possible between the cells. Pressure is controlled by maximizing the body contact area, which effects cell volume change. In this study, the height of the cushion was adjusted by the participants beforehand following the instructions in the product manual (Fig. 1-c).

\section{Wheelchair}

\section{Tilting wheelchair}

In the tilting-type wheelchair used in this study (TILT-7 [22D], Mickey Korea), the back and the seat lean back together, and the angle can be adjusted from $0^{\circ}$ to $30^{\circ}$. In this study, three angles $\left(10^{\circ}, 20^{\circ}, 30^{\circ}\right)$ were used for all participants to examine differences in pressure by angle. Before each experiment, the heights of the arm rest, foot rest, and head restraint were adjusted so that pressure measurement for all participants could be performed under the same conditions. The wheelchair is $40 \mathrm{~cm}$ wide and fitted with a backrest and head rest (Fig. 1-a).

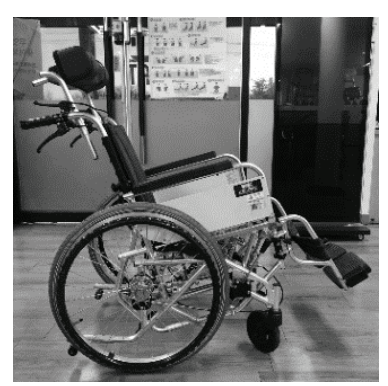

a) Tilting wheelchair

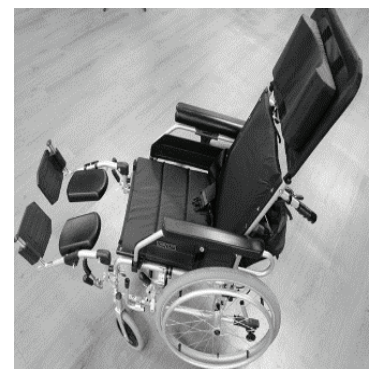

b) Reclining wheelchair

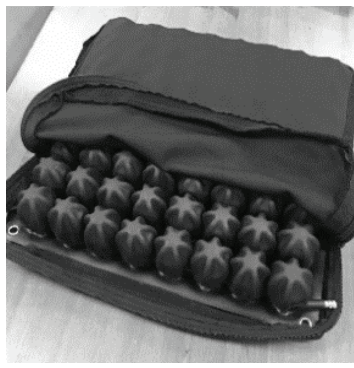

c) ROHO cushion

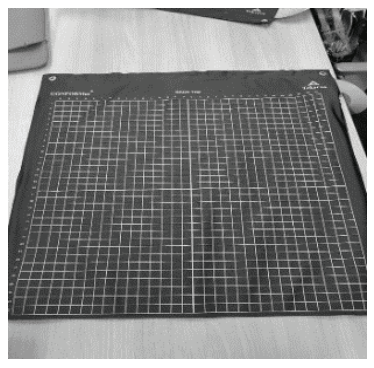

d) Pressure mapping

Figure 1. Apparatus for study 


\section{Reclining wheelchair}

The reclining wheelchair used in this study is one in which only the backrest tilts (P7003, DAESE). The backrest angle can be adjusted from $90^{\circ}$ to $180^{\circ}$. In this study, the same backrest angles were used for all subjects $\left(90^{\circ}\right.$, $110^{\circ}$, and $130^{\circ}$ ). The heights of the arm rest, foot rest, and head restraint were adjusted in advance so that pressure measurement for all participants could be performed under the same conditions. The wheelchair is $40 \mathrm{~cm}$ wide and fitted with a backrest and head rest (Fig. 1-b).

\section{Pressure mapping system}

I-Scan TM (Tekscan) was used to measure the user's hip pressure distribution when seated in the wheelchair with a ROHO cushion. I-Scan ${ }^{\mathrm{TM}}$ accurately measures and analyzes the interface pressure between two surfaces through a thin, sensitive sensor. Data can be analyzed and stored in CONFORMat Research 7.60 as pressure values (such as the average pressure, standard deviation, and maximum pressure) as well as visualized using colored contour lines. At this time, pressure was recorded in $\mathrm{mmHg}$ units, and the maximum pressure that could be measured was 200 $\mathrm{mmHg}$. The sensors can also be used as force sensors with a sampling frequency of $0-125 \mathrm{~Hz}$ (Fig. 1-d).

\section{Procedure}

Participants wore comfortable clothing without back pockets to minimize the influence of extraneous variables. Also, if necessary, the footrest height was adjusted so that the feet reached the footrest.

This study was conducted to determine the optimal angle for tilting and reclining wheelchairs when using ROHO cushions at angles of $10^{\circ}, 20^{\circ}$, and $30^{\circ}$ for tilting wheelchairs and $90^{\circ}, 110^{\circ}$, and $130^{\circ}$ for reclining wheelchairs. A pressure mapping system was used to measure the pressure, and the average pressure for each frame was measured by specifying an area with an initial value of $60 \mathrm{mmHg}$ or higher from the mid-point of both thighs to the back of the buttocks. The pressure distribution was measured for 3 minutes at each angle, with a 5-min rest period after each measurement.

\section{Data processing}

The average pressure index $(\mathrm{mmHg})$ of each frame $(50,100,150,200$ frames) was used to compare the pressure distribution according to the wheelchair (tilting and reclining) angle [7]. Because the pressure value can be adjusted according to the physical characteristics of the individual during the initial sitting, the weight and height of each subject were entered into the software to analyze changes in the pressure on and area of the cushion. In this study, the average pressure of each frame was measured, and a zone with an initial value of $60 \mathrm{mmHg}$ or more was designated.

\section{Data analysis}

The SPSS statistical package (SPSS 25.0, Armonk, NY, USA) was used to analyze the average pressure index values of all frames for the tilting- or reclining-type wheelchair used to prevent pressure sores. For general participant characteristics, descriptive statistics were used, and repeated-measures one-way ANOVA was used to compare the pressure distribution over the hip according to the backrest angle. The p-value indicating statistical significance was set to .05 . For the main effect, Bonferroni's correction was applied to identify significant differences.

\section{Results}

Pressure readings for the reclining-type wheelchair were $93.55 \mathrm{mmHg}$ at $90^{\circ}, 89.80 \mathrm{mmHg}$ at $110^{\circ}$, and $88.29 \mathrm{mmHg}$ at $130^{\circ}$. Readings for the tilting-type wheelchair were $91.91 \mathrm{mmHg}$ at $10^{\circ}, 88.53 \mathrm{mmHg}$ at $20^{\circ}$, and $86.86 \mathrm{mmHg}$ at $30^{\circ}$. The average pressure for the reclining wheelchair was lowest at $130^{\circ}$, and that for the tilting wheelchair was lowest at $30^{\circ}$. A comparison of overall pressure revealed a distribution of lower pressure using a tilting wheelchair compared to using a reclining wheelchair.

The repeated-measures ANOVA comparing pressure differences according to the angle of the backrest for each

Table. 1. Pressure distribution in recline angles $(\mathrm{N}=28)$

\begin{tabular}{lcccc}
\hline Conditions & & AI $(\mathrm{mmHg})$ & SD & F \\
\hline \multirow{3}{*}{ Reclining } & $90^{\circ}$ & 93.55 & 7.53 & \\
& $110^{\circ}$ & 89.80 & 6.38 & \\
& $130^{\circ}$ & 88.29 & 5.39 & 16.535 \\
Tilting & $10^{\circ}$ & 91.92 & 7.81 & $0.000^{*}$ \\
& $20^{\circ}$ & 88.53 & 6.42 & \\
& $30^{\circ}$ & 86.86 & 5.48 & \\
\hline
\end{tabular}




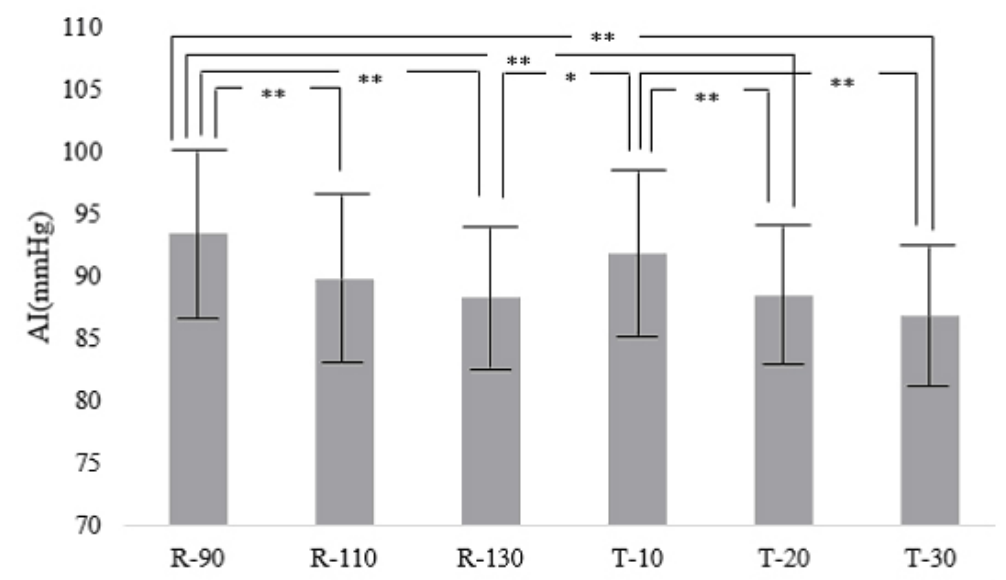

Figure 2. Pressure in the reclining and tilting wheelchair conditions $(\mathrm{N}=28$, Mean $\pm \mathrm{SD})$

R-90: 90 degree reclining wheelchair condition, R-110: 110 degree reclining wheelchair condition, R-130: 130 degree reclining wheelchair condition, T-10: 10 degree tilting wheelchair condition, T-20: 20 degree tilting wheelchair condition, T-30: 30 degree tilting wheelchair condition; $*-\mathrm{p}<0.05, * *-\mathrm{p}<0.01$ (Bonferroni-corrected).

type of wheelchair using a wound-prevention cushion revealed the following. The comparison of the average pressure index between reclining- and tilting-type wheelchairs had an F-value of 16.353, indicating a significant difference in the sitting pressure index according to wheelchair type $(\mathrm{p}=0.000)($ Table 1$)$. The multiple comparisons test showed that the pressure under the $90^{\circ}$ reclining condition was significantly higher than that at $110^{\circ}$ or $130^{\circ}$ as well as that at $20^{\circ}$ or $30^{\circ}$ when a tilting wheelchair was used $(p<0.01)$. Compared with use of the reclining-type wheelchair at $110^{\circ}$, there was no significant difference $(p>0.05)$ except at $90^{\circ}(\mathrm{p}<0.01)$. At $30^{\circ}$, the pressure from using the reclining-type wheelchair was significantly different from that at $90^{\circ}(\mathrm{p}<0.01)$ or from using the tilting wheelchair at $10^{\circ}(\mathrm{p}<0.05)$, but no significant differences were found in the other comparisons $(p>0.05)$. Significant differences were observed when the tilting wheelchair was used at $10^{\circ}$ compared to $20^{\circ}$ or $30^{\circ}$ or using the reclining wheelchair at $130^{\circ}(\mathrm{p}<0.01)$. There was no statistically significant difference between using the tilting wheelchair at $20^{\circ}$ or $30^{\circ}$ (p > 0.05) (Fig. 2).

\section{Discussion}

The purpose of this study was to determine the optimal angle of each wheelchair type by checking the effects of wheelchair type (tilting versus reclining) and backrest angle on sitting contact pressure using a ROHO pneumatic bedsore-prevention cushion to prevent pressure sores. The results showed that the average sitting pressure in the tilting-type wheelchair was lowest at $30^{\circ}$, and that in the reclining-type wheelchair was lowest at $130^{\circ}$. A comparison of total pressure showed that the pressure was lower for the tilting wheelchair than for the reclining wheelchair. Furthermore, angles of $110^{\circ}$ or more for the reclining wheelchair and $20^{\circ}$ or more for the tilting wheelchair had a significant pressure-reducing effect. However, no significant difference was found between using a reclining wheelchair at $110^{\circ}$ or $130^{\circ}$ or between using a tilting wheelchair at $20^{\circ}$ or $30^{\circ}$.

An optimal wheelchair angle adjustment may help to prevent pressure sores, and adjusting the angle according to the characteristics of the wheelchair may further reduce the risk. However, additional cushions applied to the seat can have a variety of effects depending on the user's posture. In addition, the proper use of the two means of adjustment presented in this study can be more effective than the use of a single method and an effective means of ensuring user satisfaction and health status $[4,12]$.

To determine the optimal angles for reducing pressure when using two types of wheelchairs, this study referred to past research evaluating angles of $10^{\circ}, 20^{\circ}$, and $30^{\circ}$ for tilting wheelchairs and $90^{\circ}, 110^{\circ}$, and $130^{\circ}$ for reclining wheelchairs. In a previous study, a reclining wheelchair with a pressure sore-prevention cushion was used to compare postural changes and pressure distributions between two angles $\left(90^{\circ}\right.$ and $\left.110^{\circ}\right)$ [1]. The mean pressure index was significantly lower at $110^{\circ}$ than at $90^{\circ}$. Chen and colleagues explained the necessity of adjusting the backrest angle to prevent bedsores, suggesting that the angle of the reclining chair should be set to $>120^{\circ}$ and that of the tilting chair to $>25^{\circ}[4,22]$.

In this study, two types of wheelchairs were examined while varying the angle of use, and the contact pressure on the buttocks was measured. The pressure average index (AI) was used to measure the sitting pressure distribution. 
The pressure AI of the seating surface serves as an index of the uniform dispersion of contact pressure [8].

Analysis of the average sitting pressure associated with the use of each wheelchair with an air cushion according to the pressure AI of the seating surface revealed a distribution of lower pressure for the reclining wheelchair at $110^{\circ}$ and $130^{\circ}$ compared to $90^{\circ}$. This result was partially consistent with a previous study, with some differences. As the reclining wheelchair angle changed, Jan and colleagues compared the pressure distribution over the gluteal area in patients with a spinal cord injury. A statistically significant difference was found between $100^{\circ}$ and $120^{\circ}$ [11]. In addition, Kwon and colleagues compared the average pressure change according to the reclining wheelchair angle in 20 healthy 20 -year-old men from a neutral posture of $90^{\circ}$. There were no differences at $100^{\circ}$ and $110^{\circ}$, but a large difference was observed at $120^{\circ}[4,19]$. Previous studies have demonstrated that pressure can be reduced by maintaining an angle of $120^{\circ}$ or more, but this study confirmed a significant pressurereducing effect even at $110^{\circ}$. In this study, to minimize the differences in the subjects' physical condition, their physical condition was determined based on standard values for men in their $20 \mathrm{~s}$. This study design eased the process of drawing distinctions according to wheelchair type and angle [24, 25].

For the tilting wheelchair, a significant pressure difference was observed when the pressure distribution was maintained by a tilt angle of $>20^{\circ}$. Fujita and Mori [7] compared the pressure distribution in healthy adult men to that in individuals in wheelchairs at angles of $5^{\circ}, 10^{\circ}$, $20^{\circ}$, and at full tilt. Their results showed that the contact area increased and the pressure decreased as the angle decreased. Jan and colleagues compared the pressure differences at $15^{\circ}, 25^{\circ}$, and $35^{\circ}$ when using a tilted wheelchair with a basic foam cushion in patients with a spinal cord injury. Wheelchair angles of $<25^{\circ}$ were found to be insufficient for effective pressure reduction compared with $100^{\circ}$ [11]. Our findings are partially consistent with previous studies but differ from recent findings showing that angle adjustments of $>20^{\circ}$ do not result in statistically significant changes. In the present study, a tilt angle of $20^{\circ}$ significantly decreased the pressure, although angle adjustments to $\geq 20^{\circ}$ did not have a significant effect. In this study, the tilt angle was limited to $30^{\circ}$. The reason may be that the present study was conducted mainly in the range where effectiveness had been demonstrated in previous studies. Improper use of the cushion, which may result in the user slipping down in the chair, may create the highest average maximum pressure in a sitting position. No slippage due to the cushion was observed in our study, but it is important to remember the correct backrest position to prevent unnecessary slippage when working to prevent pressure sores [3].
The ROHO cushion used in this study is relatively effective at preventing bedsores. However, few studies have considered both the angle of the cushion and that of the wheelchair back, as we did in this study. Many studies have compared cushion types with the backrest fixed at a specific angle or have changed only the angle of the backrest without using a cushion $[1,4,11]$. Incorrect posture adjustment can be accompanied by higher pressure. The placement of pillows or special positioning cushions designed to prevent pressure sores can be helpful. The cushion used in this study is the most frequently used cushion to prevent bedsores. Our study also demonstrated superior pressure reduction, and unnecessary slipping did not occur.

Our study was conducted using two recommended methods, i.e., pressure sore-preventing cushions and an adjusted backrest angle, which can improve the pressure distribution in wheelchairs. The results of this study will help wheelchair users or their guardians with selecting an optimal wheelchair angle to prevent bedsores when changing the patients' posture. Patients with bedsores often cannot manage this process themselves, so they need help from others. This situation can limit their daily lives. Furthermore, based on the results of this study, it is necessary to encourage active long-term participation of wheelchair users in daily and social life considering the physiological benefits of such engagement [16-18, 27].

The limitations of this study are as follows. First, the participants were not actual wheelchair users. This study selected subjects without disabilities to ensure participant homogeneity because it was necessary to analyze the cushion and wheelchair angle, rather than the characteristics of the participants, as the main variables. However, further studies that examine these variables will need to be planned. Second, the wheelchair posture employed in this study was a static one. As the proportion of situations in which users maintain a dynamic posture rather than a static one is high, it will be necessary to consider the difficulties associated with long-term sitting in future studies. Third, only buttock pressure was considered when the pressure distribution was measured according to the angle of the wheelchair. Thus, we did not examine how the pressure distribution affected other body parts.

\section{Conclusion}

The basic principle of preventing pressure sores is to reduce constant pressure during sitting in the wheelchair. Our study examined the optimization of two methods, the use of a pressure sore-preventing cushion and adjustments to the backrest angle. We showed that a position of $\geq 110^{\circ}$ for the reclining wheelchair and $\geq 20^{\circ}$ for the 
tilting chair had significant pressure-reducing effects. Future studies will be needed to confirm the pressure change at various angles by applying equipment that can reflect the kinematic characteristics of the subject in addition to the pressure.

\section{Conflict of interest: Authors state no conflict of interest.}

\section{References}

1. An N., Gong J.Y. (2011) Comparison of cushions for prevention of pressure ulcers and backrest angle on seated pressure. J. Korean Soc. Occup. Ther., 19: 105-115.

2. An N.Y., Joo J., Song G.H. (2010) Comparison of Cushion for Prevention of Pressure Ulcer on Seated Pressure. J. Assist. Technol., 4: 45-55.

3. Chang Y., Kim Y.C., Nam K.T., Kang J.S., Jeong B.R., Hong E. (2020) Analysis of Body Slip and Seat Pressure in Powered Wheelchairs with Two-Slide Recline System. Int. J. Precis. Eng. Manuf., 21(3): 525-535.

4. Chen Y., Wang J., Lung C., Yang T.D., Crane B.A., Jan Y. (2014) Effect of tilt and recline on ischial and coccygeal interface pressures in people with spinal cord injury. Am. J. Phys. Med. Rehabil., 93(12): 1019-1030.

5. Cho H.S., Ryu J.C., Kim G.S., Mun M.S., Lee I.H. (2007) Analysis of Body Pressure Distribution Characteristics According to The Design Factors of The Air-Cell Mattress for Preventing Decubitus Ulcer. J. Korean Soc. Precis. Eng., 24(5): 118-126.

6. Deems-Dluhy S.L., Jayaraman C., Green S., Albert M.V., Jayaraman A. (2017) Evaluating the functionality and usability of two novel wheelchair anti-rollback devices for ramp ascent in manual wheelchair users with spinal cord injury. $P M \& R, 9(5)$ : 483-493.

7. Fujita D., Mori A., Cleminson T., Kada M., Fukuda J., Kobara K., Osaka H., Watanabe S. (2010) Using seating techniques as a preventative measure against lower limb edema-the effect of combining tilt angle and reclining mechanisms on wheelchairs. J. Phys. Ther. Sci., 22(4): 437-441.

8. Giesbrecht E.M., Ethans K.D., Staley D. (2011) Measuring the effect of incremental angles of wheelchair tilt on interface pressure among individuals with spinal cord injury. Spinal Cord, 49(7): 827-831.

9. Hwangbo S., Kim J. (2015) Pressure Comparison of New Technologies of Pressure Ulcer Preventive Wheelchair Cushion : A Pilot Study. J. Korean Soc. Assist. Technol., 7(1): 1-10.

10. Jan Y., Brienza D. (2006) Technology for pressure ulcer prevention. Top Spinal Cord Inj. Rehabil., 11(4): 30-41.

11. Jan Y., Jones M.A., Rabadi M.H., Foreman R.D., Thiessen A. (2010) Effect of wheelchair tilt-in-space and recline angles on skin perfusion over the ischial tuberosity in people with spinal cord injury. Arch. Phys. Med. Rehabil., 91(11): 1758-1764.

12. Jan Y., Liao F., Jones M.A., Rice L.A., Tisdell T. (2013) Effect of durations of wheelchair tilt-in-space and recline on skin perfusion over the ischial tuberosity in people with spinal cord injury. Arch. Phys. Med. Rehabil., 94(4): 667-672.

13. Kang J.S., Chang Y.H., Jeong B.R., Kim Y.C., Hong E.P, Ryu J.C. (2016) Analysis of Trunk Slip affected by Physical Conditions when using a Recline Wheelchair. J. Korean. Soc. Precis. Eng., 875-876.

14. Kang Y.S., Yang S.H., Cho M.S. (2010) Comparative Study on the Contact Pressure Distribution of Wheelchair Seat Cushion. J. Spec. Educ. Rehab., 49(1): 183-196.

15. Kim J.H., Kim J.B. (2016) Customized Pressure Ulcer Preventive Cushion Design Research for People with Wheelchair Using. Korea Science \& Art Forum, 24: 107-117.

16. Kim S.E., Song B.S. (2015) Proposition of Korean Type Powered Wheelchair Seat Standards According to Age of the Human Scale. J. Rehabil. Welfare Eng. Assist. Technol., 9(3): 237-243.

17. Kong J.Y., An N.Y. (2009) Effect of Wedge Height of Inner on Seated Pressures. J. Spec. Educ. Rehab., 48(4): 101-118.

18. Kwon H.C., Kon J.Y. (2005) Characteristics of the buttock interface pressure according to wheelchair propulsion speed and various back reclined seating position. Phys. Ther. Korea, 12(2): 1-10.

19. Lung C.W., Yang T.D., Crane B.A., Elliott J., Dicianno B.E., Jan Y.K. (2014) Investigation of peak pressure index parameters for people with spinal cord injury using wheelchair tilt-in-space and recline: methodology and preliminary report. Biomed. Res. Int., 2014: 508583. DOI: 10.1155/2014/508583.

20. Moore Z.E., van Etten M.T., Dumville J.C. (2016) Bed rest for pressure ulcer healing in wheelchair users. Cochrane Database Syst. Rev., (10). DOI: 10.1002/14651858. CD011999.pub2.

21. Seo W., An K., Song M., Kwak S., Ko S., Jang K., Kim J. (2013) Guidelines for ulceration preventing cushions : focusing on gel and gel-air types. HCI Soc. Korea, 927-930.

22. Shankar S., Mortenson W.B., Wallace J. (2015) Taking control: an exploratory study of the use of tilt-in-space wheelchairs in residential care. Am. J. Occup. Ther., 69(2): 6902290040p1-6902290040p8

23. Size Korea (2015). Korean human body size survey. Retrieved November 19, 2019, from https://sizekorea.kr/ page/report/1.

24. Visagie S., Mlambo T., Van der Veen J., Nhunzvi C., Tigere D., Scheffler E. (2016) Impact of structured wheelchair 
services on satisfaction and function of wheelchair users in Zimbabwe. AOSIS, 5(1).

25. Ward A.L., Hammond S., Holsten S., Bravver E., Brooks B.R. (2015) Power wheelchair use in persons with amyotrophic lateral sclerosis: changes over time. Assist. Technol., 27(4): 238-245.

26. Woo J., Kim J. (2017) Intervention of Powered Seating Function : A Systematic Review. J. Korean Soc. Occup. Ther., 25(4): 33-44.

27. Yoo M., Yi M. (2015) A Phenomenological Study on Illness Experience of Patients with Pressure Ulcer. Korean J. Adult Nurs., 27(5): 515-526.
Received 28.10.2020

Accepted 04.02.2021

(C) University of Physical Education, Warsaw, Poland

\section{Acknowledgments}

I would like to thank the co-authors who took the time to provide information for the study. We would like to thank the professors at the Department of Occupational Therapy in Jeonju University who provided insight and expertise that greatly assisted this study. 